\title{
Low energy reactions of halo nuclei
}

\author{
Ismael Martel ${ }^{1 *}$ \\ ${ }^{1}$ Departamento de Ciencias Integradas, Universidad de Huelva, 21071 Huelva, Spain
}

\begin{abstract}
Halo nuclei are extreme nuclear states consisting of one or more weakly-bound valence nucleons spatially decoupled from a tightly bound nuclear core. The weakly bound nature of the halo dominates the reaction probability, but the specific reaction mechanisms depend also on the core and target nuclei. Despite of the inherent complexity of the reaction process, simple two-body models and direct reaction theories can be used to extract useful information of the structure of the halo nucleus and its dynamics. These ideas are discussed using selected experiments of Coulomb barrier reactions with one- and two- neutron halo systems.
\end{abstract}

\section{Introduction}

Nuclear systems, such as ${ }^{6} \mathrm{He},{ }^{11} \mathrm{Li},{ }^{11} \mathrm{Be}$, and ${ }^{15} \mathrm{C}$ are known to have an extended neutron distribution, the socalled neutron halo [1]. The appearance of haloes is strongly correlated with the existence of weakly bound valence nucleons, which increases the tunnelling probability through the effective nuclear potential. This effect tends to de-localise the valence nucleons, extending the nuclear density up to large distances and producing the so-called "neutron halo". Among the peculiar properties of this exotic systems, there are the appearance of soft dipole modes, with a pronounced maximum in the dipole strength $\mathrm{B}(\mathrm{E} 1)$ close to the neutron breakup threshold, very narrow transverse momentum distributions and large interaction crosssections [2]. Known halo systems accommodate at most one bound state, and often form three-body Borromean systems, like ${ }^{6} \mathrm{He}$ and ${ }^{11} \mathrm{Li}$, where none of the subsystems obtained by removing one of the valence neutrons happens to be bound.

Coulomb barrier energies (CBE) are interesting to study halo dynamics as the relative velocity is comparable to the nucleon motion in the impinging nuclei, which enhances the correlation between the relative motion and the internal degrees of freedom. Strong couplings can be induced between the elastic channel and the inelastic, transfer, breakup and fusion reactions and, for halo nuclei, the states lying in the continuum play a very important role [3]. Therefore, CBE provide a good energy range to probe the influence of the nuclear halo on reaction dynamics, testing few-body nuclear models and investigating the role of nucleon-nucleon correlations.

The elastic scattering is a peripheral process, and therefore the angular distribution of the elastic cross sections can be used to probe the extended tail of the halo wave function. The contributions of the couplings to the relevant reaction channels, often due to the dipole component of the interaction (dipole polarizability) produce a strong reduction of the elastic yield around the

\footnotetext{
* Corresponding author: imartel@uhu.es
}

grazing angle, leading to the disappearance of the Coulomb rainbow, a typical feature of the scattering of stable nuclei. At CBE the elastic channel exhibits large yields, so the measurement of the angular distribution of the elastic cross section can be an important tool to get a first information of halo systems. Values down to few tenths of mb can be measured with radioactive beams with intensities down to $10^{4}$ pps on target.

\section{Scattering of ${ }^{6} \mathrm{He}$}

The exotic nucleus "He has halo "Borromean" structure with a core of ${ }^{4} \mathrm{He}$ and two valence nucleons and has no bound states. It is by far the most investigated halo nucleus at $\mathrm{CBE}$, and more than fifty data sets are currently available at the EXFOR data base.

Our collaboration has investigated the dynamics of the system ${ }^{6} \mathrm{He}+{ }^{208} \mathrm{~Pb}$ in a wide range of energies around the barrier, from $14-22 \mathrm{MeV}$ at the Cyclotron Research facility at Louvain-la-Neuve, Belgium $[4,5]$. The large $\mathrm{Z}=82$ of the lead target generates an intense Coulomb field, strongly repelling the ${ }^{4} \mathrm{He}$ core. Thus, considering the low CBE projectile velocities, the two neutrons are dragged through the trajectory by the core with essentially only the strong (attractive) nuclear force. When approaching the target this effect will distort the shape of the ${ }^{6} \mathrm{He}$ density distribution, provoking the excitation of the low- lying states of the continuum. As a result, strong couplings are induced between the elastic channel and breakup and transfer channels, mainly due to the dipole component involved in the deformation process. On the experimental side, this effect can be observed in the shape of the angular distribution of the elastic cross sections and its correlation with the yields of ${ }^{4} \mathrm{He}$ arising from breakup and neutron transfer mechanisms.

Figure 1a shows with black dots the measured angular distributions of the elastic scattering over Rutherford at the lab energy of $22 \mathrm{MeV}$, just above the Coulomb barrier $(\sim 20 \mathrm{MeV})[4,5]$. It is observed a strong absorption pattern up to very small scattering angles 
down to $\theta \sim 40^{\circ}$, much below the grazing angle expected at $\theta \sim 110^{\circ}$. In semiclassical approach this corresponds to a very large projectile-target distance-of-closest approach as far as $r_{\max }=22 \mathrm{fm}$, which brings out the appearance of long-range reaction mechanisms (this is more clearly shown in the data of Figure $1 \mathrm{~b}$, which includes also intermediate scattering angles). Note the disappearance of the nuclear rainbow, a typical signature of the heavy ion scattering found in stable nuclei such e.g., ${ }^{4} \mathrm{He}+{ }^{208} \mathrm{~Pb}$ or ${ }^{6,7} \mathrm{Li}+{ }^{208} \mathrm{~Pb}$ at $\mathrm{CBE}$.

In Figure 1a we also show three Optical Model (OM) calculations: a prediction using a volume complex potential obtained from systematic studies of stable nuclei [6] (dashed line, BOM); a fit of the data (solid line, FOM) obtained by varying this potential; a fit adding a semiclassical dipole polarization potential (dotdashed line, DPP) to include the Coulomb part of the dipole coupling [7]; and a calculation obtained by removing from the last one the Coulomb dipole coupling (NCD) (dotted line). We can see that prediction based on BOM is far from the data, resulting in a pronounced nuclear rainbow and reduced absorption, and therefore halo-nuclei cannot be described by directly extrapolating parameters obtained from systematics of stable nuclei. The data are well reproduced with the FOM calculations using a shallow imaginary potential with extremely large imaginary diffuseness $\sim 1.8 \mathrm{fm}$, about three times that of stable nuclei. This is a typical signature of CBE halo scattering, which arises from long-range reaction mechanisms. By comparison with the DPP calculation, it is seen that the effect of the Coulomb dipole part of the interaction produces only part of the absorption, mainly at large scattering angles.

The effect of the couplings on the reaction dynamics is more detailed investigated using Coupled Discretised Coupled Channel Calculations (CDCC) by including the effects of coupling to the continuum. The results are shown in Figure 1b $[8,9]$. In this case ${ }^{6} \mathrm{He}$ has been modelled as a ${ }^{4} \mathrm{He}$ core plus a di-neutron system (2n), which highlights the strong correlations among halo neutrons. Three calculations are shown: a full calculation (FC) that reproduces the data (red-solid line); a calculation (NC) obtained by removing the coupling to the continuum (purple-dashed line); and a calculation (ND) obtained by removing only the dipole component of the nuclear part of the coupling to the continuum (green-dashed line). It should be noticed that the FC can nicely reproduce the angular distribution using this simple model for ${ }^{6} \mathrm{He}$ using a standard $\mathrm{d}-{ }^{208} \mathrm{~Pb}$ potential. In fact, this $2 \mathrm{n}$ halo model can simultaneously reproduce the angular and energy distributions of the ${ }^{4} \mathrm{He}$ fragments by assuming neutron transfer to the continuum; this idea will be discussed later in some more detail. What it is interesting about the CDCC results is that although the system is dominated, as expected, by the coupling to continuum states (compare FC with NC), the nuclear part of the dipole couplings plays the dominant role, as can be seen by comparing $\mathrm{NC}$ with ND. In fact, it is the coherent interference between nuclear and Coulomb couplings that allows to reproduce the data.
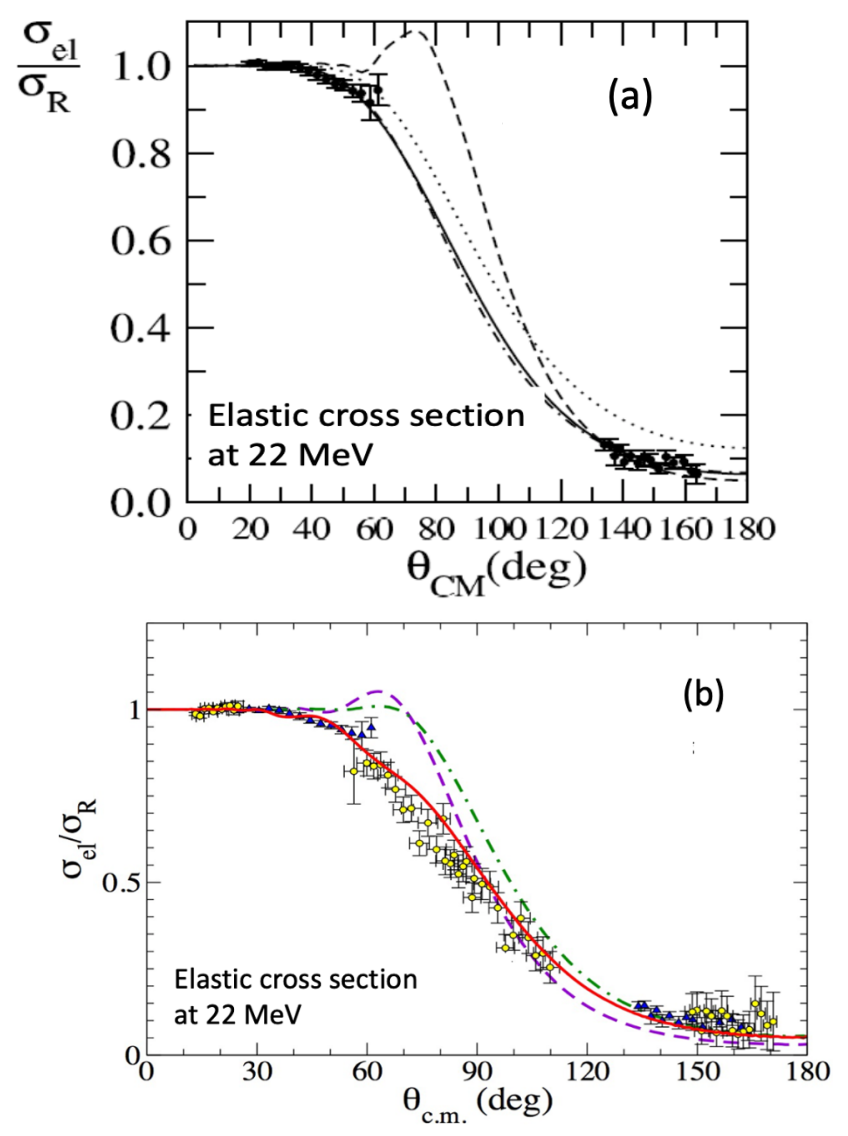

Fig. 1. Elastic scattering of the system ${ }^{6} \mathrm{He}+{ }^{208} \mathrm{~Pb}$ at $22 \mathrm{MeV}$ from ref [3] (up) and ref. [4] (down). The various curves are scattering calculations; see text for details.

The angular distributions of the ${ }^{4} \mathrm{He}$ events, normalised to the elastic scattering, is shown in Figure 2(a). We observe large ratios even at energies well below the Coulomb barrier, still about $10 \%$ at $14 \mathrm{MeV}$ collision energy, while the contribution of the Coulomb dipole component of the coupling, relative to the nuclear part, is increased [10]. Using the data at $18 \mathrm{MeV}$ a value of the polarizability of ${ }^{6} \mathrm{He}$ is found to be $1.2 \mathrm{fm}^{3}[11]$, in good agreement with that given in Ref. [12] using high energy reactions.

To further investigate the reaction mechanism, we carried out additional Distorted Wave Born Approximation (DWBA) calculations for one and two neutron stripping using the $2 \mathrm{n}$ model with binding energy $\varepsilon_{\mathrm{b}}=0.9 \mathrm{MeV}$. The result is compared with the previous CDCC calculations and the data in figure 2(b) and 2(c). The solid black and blue lines (TC) depict the calculated $2 \mathrm{n}$ transfer angular and energy distributions, the dash-dot line (NT) the single neutron stripping, and the dashed line the direct breakup distributions (DBU) calculated with CDCC. The black arrow in panel 2(c) marks the energy of $\sim(4 / 6) \mathrm{E}_{\mathrm{Lab}}$ where the events arising from direct breakup should be most probably expected. The TC calculation reproduces well both the angular and energy distribution of the ${ }^{4} \mathrm{He}$ fragments at backward angles, assuming the direct transfer of a di-neutron cluster to high excited states in ${ }^{210} \mathrm{~Pb}$ just above the twoneutron separation energy. This peculiar transfer 
process was named "transfer to the continuum". Single neutron stripping becomes relevant around $80^{\circ} \mathrm{Lab}$, whereas the direct breakup yield at much forward angles $\sim 30^{\circ} \mathrm{Lab}$. In a semiclassical picture, the CBE dynamics of ${ }^{6} \mathrm{He}+{ }^{208} \mathrm{~Pb}$ can be interpreted as a combined action of the repulsive Coulomb interaction core - target at large distances, generating breakup events, and the attractive nuclear part of the interaction producing transfer of neutrons at the vicinity of the target, close to the semiclassical turning point, with almost zero relative velocity and Q-value. A post-acceleration effect on ${ }^{4} \mathrm{He}$ fragments after the $2 \mathrm{n}$ transfer, arising from the Coulomb repulsion by the ${ }^{208} \mathrm{~Pb}$ target, can be observed.
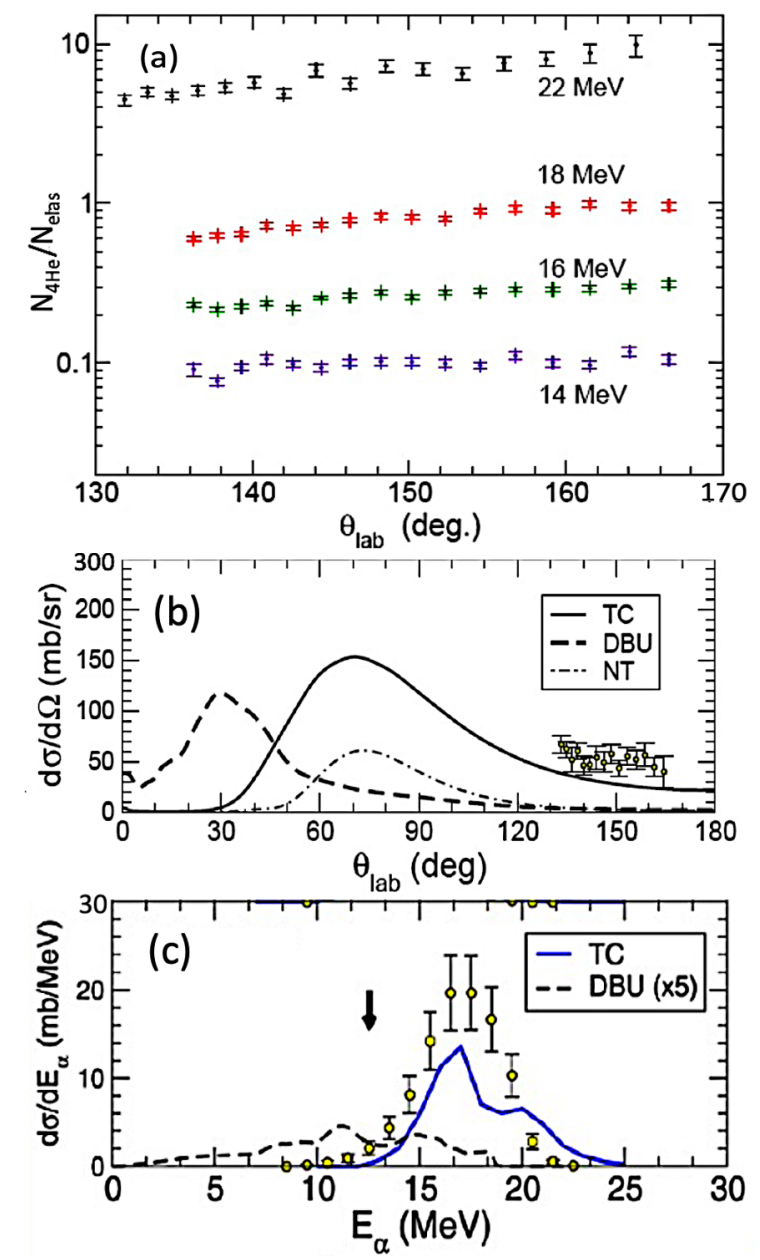

Fig. 2. (a) Angular distribution of the relative yields $\mathrm{N}_{4 \mathrm{He}} / \mathrm{N}_{\text {elas }}$ in a range of collision energies between $14 \mathrm{MeV}-22 \mathrm{MeV}$. (b) Angular distribution of the cross section for ${ }^{4} \mathrm{He}$ fragment production. (c) Energy distribution of ${ }^{4} \mathrm{He}$ fragments for the angular range $132^{\circ}-164^{\circ}$. The black arrow in panel 2(c) marks the energy of $\sim(4 / 6) \mathrm{E}_{\mathrm{Lab}}$ where the events arising from direct breakup should be expected. See text for discussion.

The CBE of ${ }^{8} \mathrm{He}$ system constitutes an ideal testbench for improving dynamic polarization potentials to describe coupling to breakup and continuum excitations $[13,14]$, and for developing di-neutron and four body models [15-17].

The vast amount of data available makes it possible to develop systematic models of CBE scattering by introducing suitable scaling parameters. Concerning the total reaction cross sections $\sigma_{\text {Reac }}$, the authors of ref. [18] find a systematic behaviour with the collision energy, by using a scaling factor for the collision energy proportional to the Coulomb-barrier height, $\mathrm{ZpxZt} /\left(\mathrm{Ap}^{1 / 3}+\mathrm{At}^{1 / 3}\right)$ and a cross-section scaling factor $\left(\mathrm{Ap}^{1 / 3}+\mathrm{At}^{1 / 3}\right)^{2}$ proportional to the size of the scattering system. The reduced cross sections $\sigma_{R}$ for ${ }^{6} \mathrm{He}$ and ${ }^{4} \mathrm{He}$ scattering is represented with respect to the reduced collision energy $\mathrm{E}_{\mathrm{Red}}$ in Figure 3, for a wide range of targets from ${ }^{12} \mathrm{C}$ to ${ }^{209} \mathrm{Bi}$. The data can be fitted using the simple analytical model of Wong [19], where the reaction cross section is calculated by the quantum tunnelling of an effective "reaction barrier" of parabolic shape. The results of the fit are shown in the figure with black solid lines.

The consequence of this study is of very much importance. It demonstrates that (1) ${ }^{6} \mathrm{He}$ reactions at CBE can be described by a "universal" reaction function, (2) the appearance of two separate halo effects, one due to the size and other due to its peculiar dynamics and (3), a clear evidence for core-halo decoupling in reactions induced by ${ }^{6} \mathrm{He}$ at $\mathrm{CBE}$. A more detailed comparison of light-ion $\mathrm{CBE}$ scattering highlighting bound, weakly-bound and halo systems is given in ref. [20].

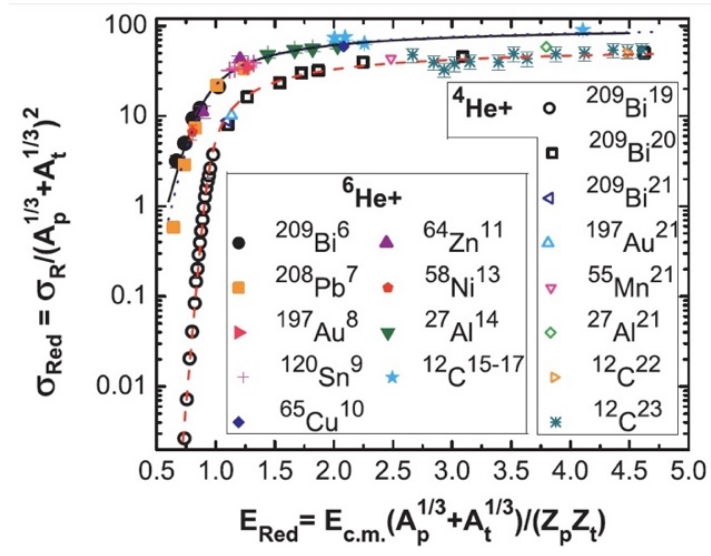

Fig. 3. Reaction cross sections for ${ }^{6} \mathrm{He}$ and ${ }^{4} \mathrm{He}$ at several collision energies and targets [19].

The regularities in the scattering observables can be further investigated by using the angular distributions of the elastic cross sections. In a semiclassical approach, the ratio between the elastic cross sections and the Rutherford cross section $P_{e l}$ can be interpreted as a probability for the occurrence of the elastic process. When plotted versus the distance of closest approach $\mathrm{r}_{\max }$ in a Coulomb trajectory, the shape of the distribution $P_{e l}\left(\mathrm{r}_{\max }\right)$ exhibits the characteristic distance at which the absorption takes place. This is shown in Figure 4 (Top) for the scattering data of ${ }^{6} \mathrm{He}+{ }^{208} \mathrm{~Pb}$ in a range of energies from 14-27 MeV, just around the Coulomb barrier [21, $22]$. The overall trend of the data, which covers several energies, can be well described by using a semiclassical model with only a diffuse imaginary proximity potential (red solid line) (i.e., no real potential) with a set of parameters similar to the complex potential obtained from the OM calculation [1]. This reveals that the collision is dominated by a very strong absorption 
starting at large distances ( $22 \mathrm{fm}$ in the figure), a signature of the presence of long-range reaction mechanisms. However, what is somehow surprising is that the data of such a wide range of energies can be described by the same potential, meaning that the absorption depends strongly on the distance of closest approach.

On the other hand, as ${ }^{6} \mathrm{He}$ is Borromean, weakly bound and has no excited states, thus any reaction should mainly lead to the production of ${ }^{4} \mathrm{He}$ fragments. In the semiclassical approach, this reaction probability can be also estimated as the ratio between the angular distribution of the cross section for ${ }^{4} \mathrm{He}$ production and the Rutherford cross section, and the result is shown in Figure 4, as a function of the distance of closest approach. The reaction probability can be estimated as $\operatorname{Pr}\left(r_{\max }\right)=1-\operatorname{Pel}\left(r_{\max }\right)$, and this is shown with a dashed black line. It is surprising the good agreement obtained, despite the fact that the trajectory of the alphas should be modified due to the loss of the two-neutrons [23]. This suggests that the dominant dynamical process is actually a "soft" neutron transfer with reduced Q-value and momentum transfer in the vicinity of the distance of closest approach, producing a small disruption on the Coulomb trajectory. On the other hand, it shows that almost all the reaction cross section is exhausted by the alpha production mechanism at large distances, leaving very little room for complete fusion. This was demonstrated in a series of experiments with ${ }^{208} \mathrm{~Pb}$ and ${ }^{238} \mathrm{U}$ targets [24].
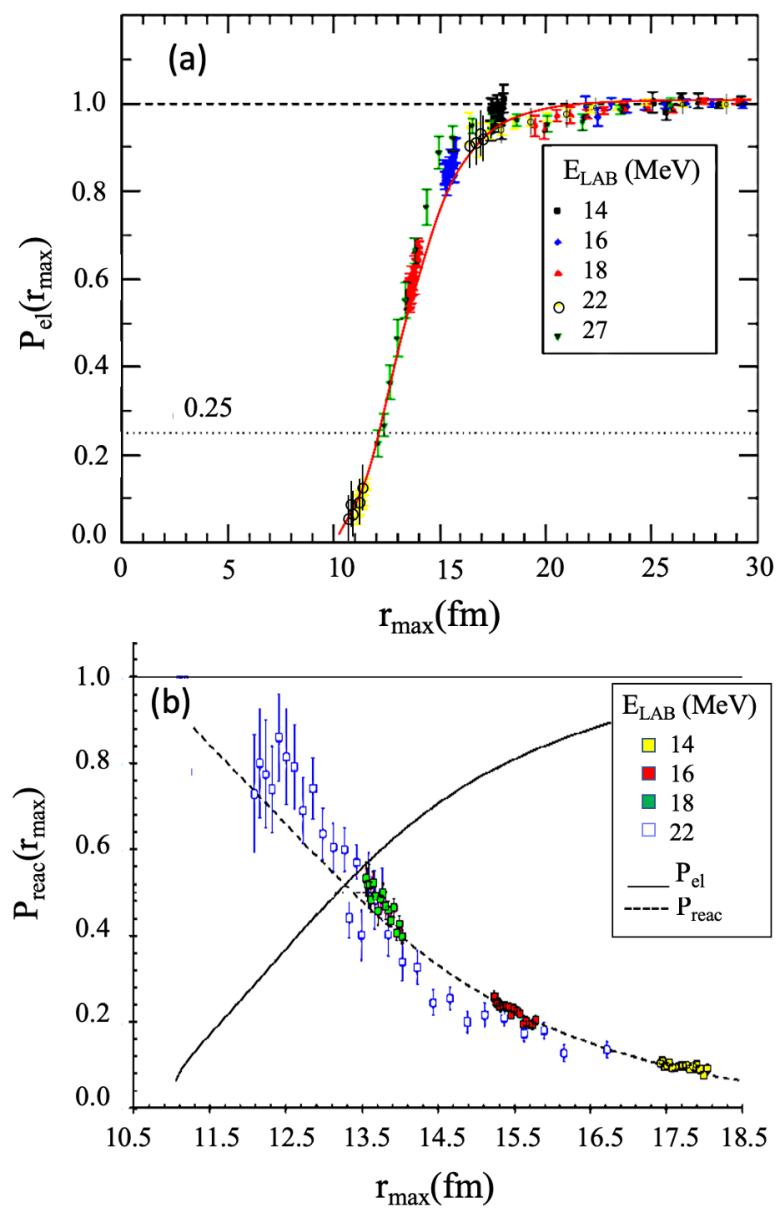

Fig. 4. (a) Ratio of the elastic cross sections over Rutherford Pel $\left(r_{\max }\right)$, as a function of the distance of closest approach in a Coulomb trajectory for the reaction ${ }^{6} \mathrm{He}+{ }^{208} \mathrm{~Pb}$. (b) Same as before for the ratio of the cross section for alpha production over Rutherford, $\mathrm{P}_{\text {reac }}\left(\mathrm{r}_{\max }\right)$.

\section{Scattering of ${ }^{8} \mathrm{He}$}

${ }^{8} \mathrm{He}$ is the most neutron-rich particle-stable nucleus with $\mathrm{N} / \mathrm{Z}=3$ and it has a Borromean neutron structure ${ }^{4} \mathrm{He}+4 \mathrm{n}$, as the system ${ }^{7} \mathrm{He}$ is unbound. The main interest is about the study of the dynamics of the neutron skin. The neutron skin is characterised by a concentration of the neutron density around the nuclear core, whereas for a neutron halo the density is diffuse and extends to large distances from the core. ${ }^{8} \mathrm{He}$ is also unique as it can produce neutron transfer and breakup to ${ }^{6} \mathrm{He}$ and ${ }^{4} \mathrm{He}$. Therefore, the CBE of ${ }^{8} \mathrm{He}$ makes possible the study the competition of multi-neutron transfer and breakup. On the other hand, the comparison of ${ }^{8} \mathrm{He}$ and ${ }^{6} \mathrm{He}$ scattering can reveal a different dynamics for skin and halo. The neutron separation energy is much higher in ${ }^{8} \mathrm{He}$ than in ${ }^{6} \mathrm{He}$, so the breakup channel should be reduced.

In Figure 5 (a) it is shown the experimental angular distribution of the elastic scattering cross section over Rutherford for ${ }^{8} \mathrm{He}$ (black filled circles) and ${ }^{6} \mathrm{He}$ (white circles) with a ${ }^{208} \mathrm{~Pb}$ target at $\mathrm{E}_{\mathrm{Lab}}=22 \mathrm{MeV}$ [25]. The solid and dashed lines are OM calculations for ${ }^{8} \mathrm{He}$ and ${ }^{6} \mathrm{He}$, respectively. Note that for ${ }^{8} \mathrm{He}$ and ${ }^{6} \mathrm{He}$, the collision energies in centre of mass $(\mathrm{Ecm})$ are $21.2 \mathrm{MeV}$ and 21.4 MeV, respectively, and the Coulomb barriers ( $\mathrm{Vb}$ ) are $23.8 \mathrm{MeV}$ and $24.4 \mathrm{MeV}$. Thus the observed differences in the angular distributions are mostly due to the dominant reaction mechanisms, and not to the kinematics. The data of ${ }^{8} \mathrm{He}$ presents a sharp fall-off around $60^{\circ}$ pointing to a localised absorption, whereas for ${ }^{6} \mathrm{He}$ the absorption starts at very small angles $\sim 45^{\circ}$ and decreases smoothly with the scattering angle. The different behaviour is more clearly seen in Figure 5 (b), where the cross sections are plotted as a function of the distance of closest approach $r_{\max }$ instead of the scattering angle. In the case of ${ }^{6} \mathrm{He}$ scattering, a $2 \%$ reduction in the cross sections is reached at distances as long as 19.5 $\mathrm{fm}$, exhibiting a smooth decay as the distance is reduced, whereas in the case of ${ }^{8} \mathrm{He}$ goes down to $16.2 \mathrm{fm}$ with a sharp absorption. The OM gives similar reaction cross sections for both isotopes $\sim 1500 \mathrm{mb}$, but in the case of ${ }^{8} \mathrm{He}$, the imaginary optical potential has much larger radius and smaller imaginary diffusivity than in the case of ${ }^{6} \mathrm{He}$.

In Figure 5a it is shown the experimental angular distribution of the elastic scattering cross section over Rutherford for ${ }^{8} \mathrm{He}$ (black filled circles) and ${ }^{6} \mathrm{He}$ (white circles) with a ${ }^{208} \mathrm{~Pb}$ target at $\mathrm{ELab}=22 \mathrm{MeV}$ [25]. The solid and dashed lines are OM calculations for ${ }^{8} \mathrm{He}$ and ${ }^{6} \mathrm{He}$, respectively. Note that for the lead target, the collision energies in centre of mass and the corresponding Coulomb barriers are very similar. Thus the observed differences in the angular distributions are 
due to the reaction mechanisms, and not to the kinematics.
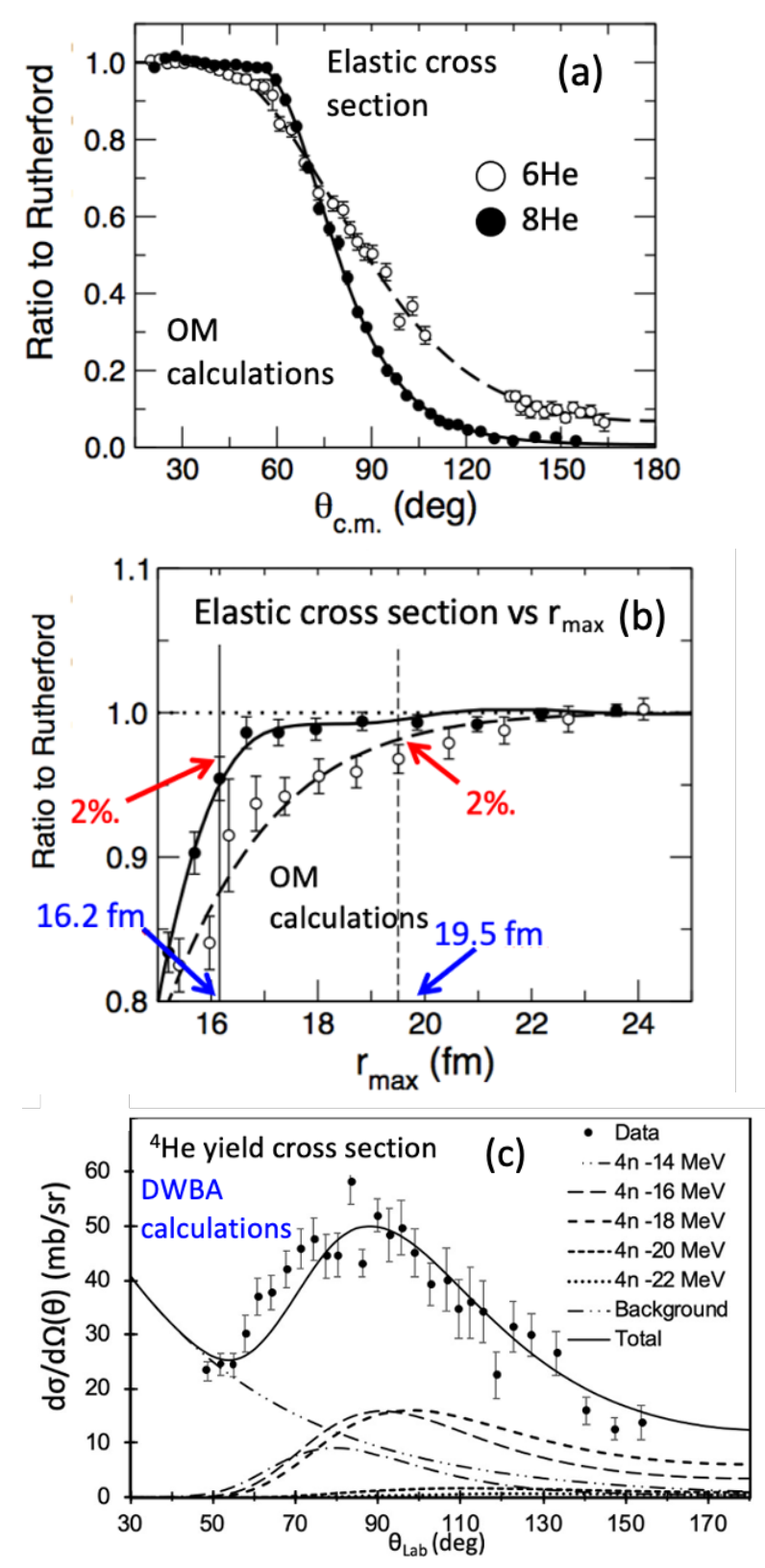

Fig. 5. Experimental angular distribution of the elastic scattering cross section over Rutherford for ${ }^{8} \mathrm{He}$ (black filled circles) and ${ }^{6} \mathrm{He}$ (white circles) with a ${ }^{208} \mathrm{~Pb}$ target at $\mathrm{E}_{\mathrm{Lab}}=22$ $\mathrm{MeV}$ [25]. The solid and dashed lines are OM calculations for ${ }^{8} \mathrm{He}$ and ${ }^{6} \mathrm{He}$, respectively. (b) As before, but as a function of the distance of closest approach $r_{\max }$. (c) Experimental angular distribution of the cross section for ${ }^{4} \mathrm{He}$ production in the scattering of ${ }^{8} \mathrm{He}+{ }^{208} \mathrm{~Pb}$ at $\mathrm{E}_{\mathrm{Lab}}=22 \mathrm{MeV}$. The data can be reproduced by DWBA calculations assuming $4 \mathrm{n}$ cluster transfer to high excited states in ${ }^{212} \mathrm{~Pb}$ by fitting the relevant spectroscopic factors [26]. The solid line is thus a fit of the data, and the other lines the partial contributions from different excited states in ${ }^{212} \mathrm{~Pb}$.

The data of ${ }^{8} \mathrm{He}$ presents a sharp fall-off around $60^{\circ}$ whereas for ${ }^{6} \mathrm{He}$ the absorption starts at very small angles $\sim 45^{\circ}$ and decreases smoothly with the scattering angle. The different behaviour is more clearly seen in Figure $5 b$, where the cross sections are plotted as a function of the distance of closest approach $r_{\max }$ instead of the scattering angle. In the case of ${ }^{6} \mathrm{He}$ scattering, a $2 \%$ reduction in the cross sections is reached at distances as long as $19.5 \mathrm{fm}$, exhibiting a smooth decay as the distance is reduced, whereas in the case of ${ }^{8} \mathrm{He}$ goes down to $16.2 \mathrm{fm}$. The $\mathrm{OM}$ gives similar reaction cross sections for both isotopes $\sim 1500 \mathrm{mb}$, but in the case of ${ }^{8} \mathrm{He}$, the imaginary optical potential has much larger radius and smaller imaginary diffusivity than in the case of ${ }^{6} \mathrm{He}$. The different behaviour can be attributed to the differences in skin and halo. In the case of the halo, the combination of the extended neutron distribution, the weak binding and the dipole polarizability make the system break from very large distances to the target, and although there is a neutron transfer mechanism occurring in the vicinity of the target, it populates highly excited levels of ${ }^{210} \mathrm{~Pb}$ lying in the continuum. In the case of ${ }^{8} \mathrm{He}$, the neutron skin is more tightly bound and localised, and therefore the breakup and neutron stripping mechanism are induced closer to the target, and the breakup is weaker due to the higher binding energy.

In a recent work [26] the cross sections and energy distribution for the ${ }^{8,6} \mathrm{He}$ fragments produced in the ${ }^{8} \mathrm{He}+{ }^{208} \mathrm{~Pb}$ reaction [25], were analysed in detail. By using DWBA calculations it is found that the neutron transfer process is dominated by (1) single neutron stripping to low excited states of ${ }^{209} \mathrm{~Pb}\left({ }^{6} \mathrm{He}\right.$ in the exit channel) and (2) the transfer of ${ }^{4} n$ clusters to excited states in ${ }^{212} \mathrm{~Pb}\left({ }^{4} \mathrm{He}\right.$ in the exit channel). The total cross sections obtained are quite large $\sim 900 \mathrm{mb}$ and $400 \mathrm{mb}$, respectively. The angular distribution of the cross sections for ${ }^{4} \mathrm{He}$ production is shown in Figure 5 (c). The experimental data are shown with black circles. The data can be well reproduced by DWBA calculations assuming the ${ }^{4} \mathrm{n}$ transfer to various excited sates in ${ }^{212} \mathrm{~Pb}$ (black solid line), by fitting the spectroscopic factors. The partial contributions of the transfer to various excited states are also shown. In order to confirm this results, it would be interesting to measure the exclusive ${ }^{6,} \mathrm{He}$ angular distributions in coincidence with gammas (and neutrons) produced in the decay of lead isotopes populated in the stripping process.

\section{Scattering of ${ }^{11} \mathrm{Be}$}

The isotope ${ }^{11} \mathrm{Be}$ is known to exhibit a single neutron halo structure with a ${ }^{10} \mathrm{Be}$ core. It also has one bound excited state at $320 \mathrm{keV}$ with largest known $\mathrm{B}(\mathrm{E} 1)$ transition strength to the ground state [27]. To explain this phenomenon it was actually noticed for first time the need to integrate the matrix elements up to large distances, presumably due to the extension of the gs wave function. The theoretical treatment of ${ }^{11} \mathrm{Be}$ might appear to be simpler as compared to Borromean systems like ${ }^{6} \mathrm{He}$ and ${ }^{8} \mathrm{He}$, but in this case the deformation of the ${ }^{10} \mathrm{Be}$ plays an important role in the reaction dynamics, which is absent in helium isotopes.

One of the first investigations of CBE dynamics using ${ }^{11} \mathrm{Be}$ beams was the system ${ }^{11} \mathrm{Be}+{ }^{209} \mathrm{Bi}$, which was measured between $35-50 \mathrm{MeV}$ at the RIKEN RIPS 
facility [28]. The idea was to study the effect of the neutron excess on the complete fusion. Due to the halo structure, ${ }^{11} \mathrm{Be}$ should exhibit a large hindrance factor as compared to the stable isotope ${ }^{9} \mathrm{Be}$. It should be also an effect in ${ }^{10} \mathrm{Be}$ due to the deformation and coupling to the first excited state.
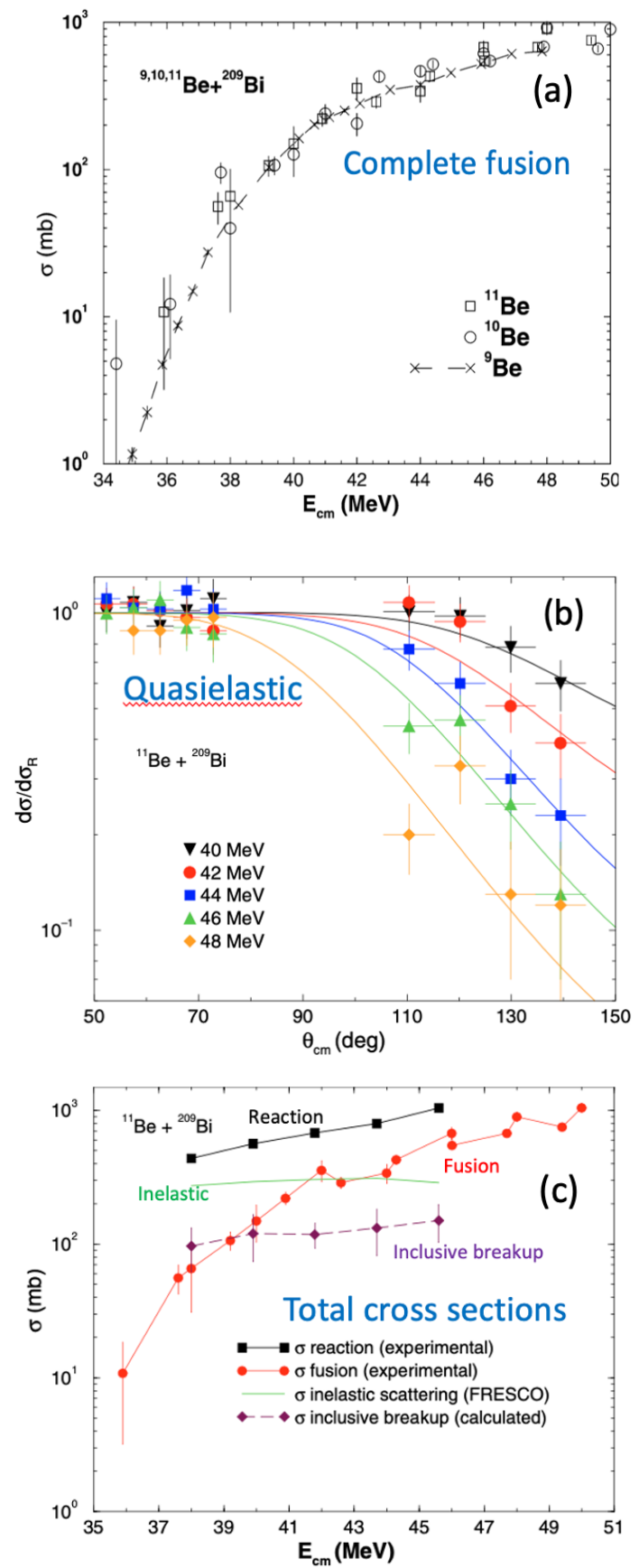

Fig. 6. (a) Fusion cross sections of the isotopes ${ }^{11,10,9} \mathrm{Be}$ with a ${ }^{209} \mathrm{Bi}$ target [28]. (b) Angular distribution of the quasielastic cross sections for ${ }^{11} \mathrm{Be}+{ }^{209} \mathrm{Bi}$ for $\mathrm{E}=40-48 \mathrm{MeV}$ [29]. (c) Energy dependence of the reaction cross sections, fusion, inelastic and breakup. See text for discussion.

The results of the experiment, shown in Figure 6 (a), were quite puzzling, as they found almost identical energy distribution of the complete fusion for the three isotopes, except at very deep sub-barrier energies, where the data showed a slightly larger fusion cross sections for ${ }^{11} \mathrm{Be}$ than ${ }^{9} \mathrm{Be}$ that could be attributed to a halo effect. The data could be reproduced using CCFULL and
CDCC calculations, which suggested a strong role of the breakup channel in the suppression of complete fusion for the halo system ${ }^{11} \mathrm{Be}$. The halo would break at large distances before reaching the target, reducing the fusion probability. This was also observed in the case of ${ }^{6} \mathrm{He}$ [24]. The system ${ }^{11} \mathrm{Be}+{ }^{209} \mathrm{Bi}$ was also investigated in [29], where the angular distribution of the quasielastic cross sections was measured between $40-48 \mathrm{MeV}$. The result is shown in Figure $6 \mathrm{~b}$. The data were well described by OM, DWBA and Coupled-Channel calculations, assuming a rotational model with a deformation parameter obtained from the experimental $\mathrm{B}(\mathrm{E} 1)$ value.

Using the measured reaction cross sections, the calculated inelastic cross sections and the fusion cross sections of the previous experiment [28], the energy distribution of the total cross sections for breakup could be deduced. The results are shown in Figure $6 \mathrm{c}$. The reaction cross sections were quite large $\sim 800 \mathrm{mb}$, about ten times the fusion cross sections; however the calculated breakup cross sections were relatively small $\sim 100-150 \mathrm{mb}$, even below the fusion yield, which was somehow surprising for such a weakly bound halo system.

The $\mathrm{CBE}$ of ${ }^{11} \mathrm{Be}$ was later investigated by using a ${ }^{120} \mathrm{Sn}$ at $\mathrm{E}_{\mathrm{Lab}}=32 \mathrm{MeV}$ at the RIB facility REX-ISOLDE at CERN, where the angular distribution of both quasielastic and ${ }^{10} \mathrm{Be}$ fragments were measured for the first time [30]. The angular distribution of the quasielastic cross section is shown in Figure 7a (black dots) and is compared with the scattering of ${ }^{9} \mathrm{Be}+{ }^{120} \mathrm{Sn}$ at similar collision energy [31] (yellow circles). The Coulomb-nuclear interference is strongly damped and the deviation from Coulomb occurs around $\sim 30^{\circ} \mathrm{cm}$, a clear signature of the neutron halo, and the presence of long-range reaction mechanisms.

The dynamics was also investigated with $\mathrm{CC}$ calculations using a simple vibrational model for ${ }^{11} \mathrm{Be}$ (deformation length derived from experimental $\mathrm{B}(\mathrm{E} 1)$, including the coupling to the bound excited state at 320 $\mathrm{keV}$ and the first two resonances at $1.78 \mathrm{MeV}$ and 3.41 $\mathrm{MeV}$, and two "fictitious" dipole states at $\varepsilon=0.55 \mathrm{MeV}$ and $I=1 / 2-, 3 / 5-$, just above the neutron separation energy, to simulate the effect of the dipole couplings to the continuum. The calculation reproduces well the quasielastic angular distribution. The effect of removing the coupling to the "fictitious" dipole states is shown by the red-dotted and blue-dashed lines. The former contains only the coupling to the 1 st excited state, and the latter adding the resonances with negligible effect. This angular distributions are very similar to the experimental angular distribution of the stable nucleus ${ }^{9} \mathrm{Be}$ (yellow circles). This result brings out the importance of the coupling to the low-energy continuum dipole states in the CBE dynamics of ${ }^{11} \mathrm{Be}$. On the other hand, although the cross section for excitation is relatively large, the effect of the coupling has a small effect on the shape of the angular distribution of the quasielastic cross section. 

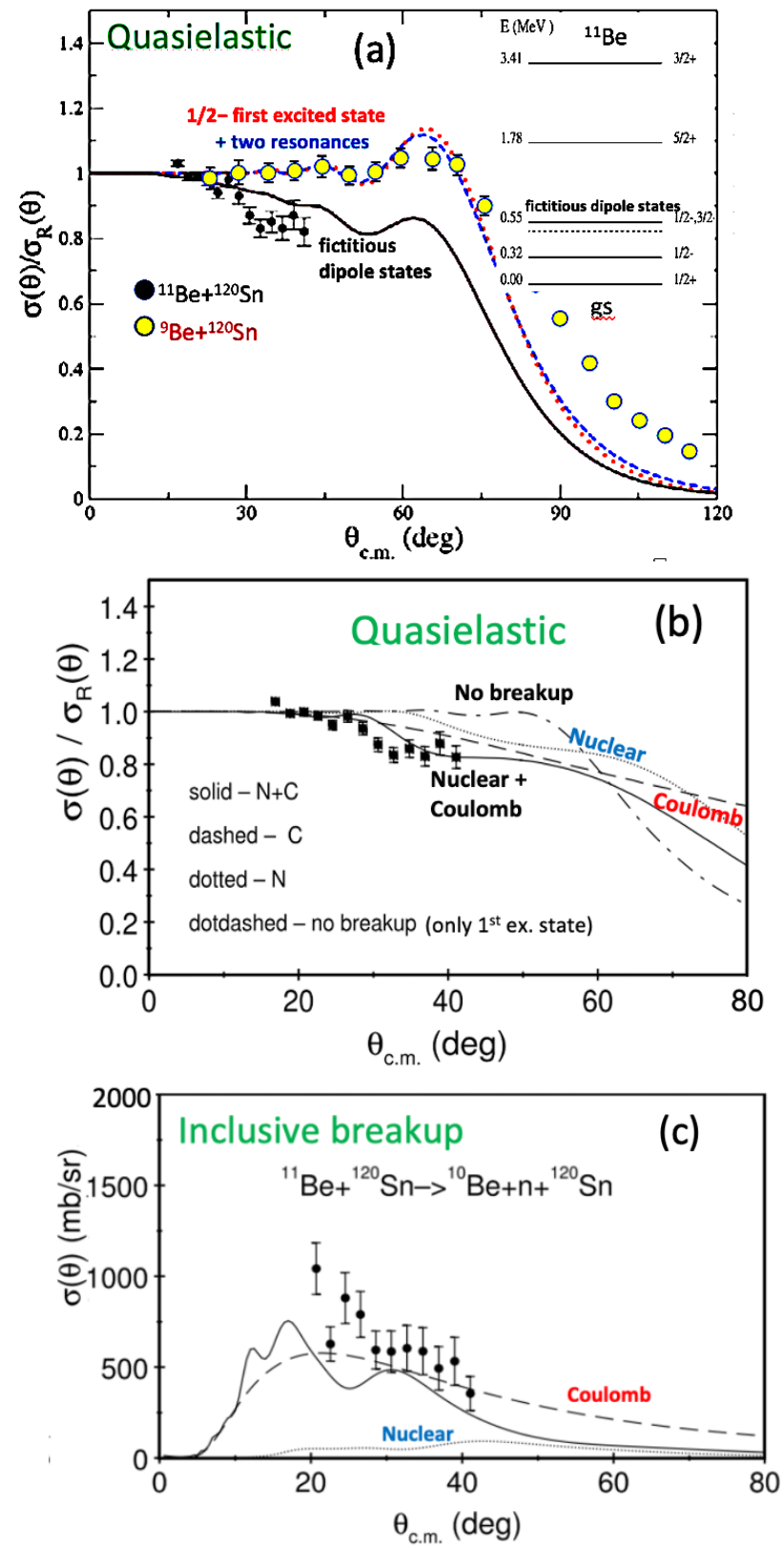

Fig. 7. (a) Angular distribution of the quasielastic cross sections for ${ }^{11} \mathrm{Be}+{ }^{120} \mathrm{Sn}$ at $\mathrm{E}=32 \mathrm{MeV}$ [30] (black circles), and for the system ${ }^{9} \mathrm{Be}+{ }^{120} \mathrm{Sn}$ at similar collision energy. (b) CDCC calculations are compared with the experimental quasielastic cross section (black squares) [31]. (c) Angular distribution of the breakup cross sections. Various calculations are shown with solid, dashed and dotted lines; see text for explanation.

The coupling to the continuum is investigated with more detail in [32] using CDCC calculations. In Figure 7b the calculations (solid black line, $\mathrm{N}+\mathrm{C}$ ) are compared with the data of the quasielastic cross sections (black circles). The dot-dashed line is a calculation obtained by removing the coupling to the continuum leading to breakup but keeping the coupling to the 1 st excited state. This demonstrates, as just discussed, that the dynamics is dominated by dipole couplings to the continuum. The dashed line (C) results from removing the Coulomb dipole couplings and the dotted line $(\mathrm{N})$ from removing the nuclear dipole couplings. A strong Coulombnuclear interference effect is observed where Coulomb and nuclear contributions interfere destructively.
In Figure $7 \mathrm{c}$ the predictions for breakup cross sections (solid black line) are compared with the experimental data (black circles). The calculation reproduces well the shape of the data, with a total breakup yield about $50 \%$ of the total cross sections. The dipole Coulomb coupling produces most of the breakup yield (dashed line) and the nuclear component is small (dotted line).

Although the Coulomb dipole coupling to the continuum dominates the reaction dynamics, the nuclear part plays a non-trivial role due to coherent interference effects, which are important to reproduce the measured angular distribution of both quasielastic and breakup. Another interesting feature arises from the energy distribution of ${ }^{10} \mathrm{Be}$ fragments, where the effect of Coulomb post-acceleration can be observed with an energy shift of $\sim 1.8 \mathrm{MeV}$ [32] suggesting breakup taking place at distances $\sim 15 \mathrm{fm}$ from the target. This is consistent with the long-rage absorption mechanisms.

A more recent experiment performed at REXISOLDE/CERN was the CBE of the system ${ }^{11} \mathrm{Be}+{ }^{64} \mathrm{Zn}$ at $\mathrm{E}=28.7 \mathrm{MeV}[34,35]$ where the angular distribution of the cross sections for quasielastic and ${ }^{10} \mathrm{Be}$ fragments (breakup) were measured. The use of the smaller $\mathrm{Z}=30$ target ${ }^{64} \mathrm{Zn}$ allows a deeper access to the nuclear part of the nuclear interaction producing coupling to the continuum and breakup. The angular distribution of the quasielastic scattering cross sections is shown in Figure $8 \mathrm{a}$ using black squares and is compared with the scattering of non-halo isotopes ${ }^{10} \mathrm{Be}$ and ${ }^{9} \mathrm{Be}$ at the same centre of mass collision energy. As in the previous case, as compared to the stable isotope ${ }^{9} \mathrm{Be}$, the Coulombnuclear interference is strongly damped and there is a clear deviation from Coulomb scattering from about $\theta \sim$ $25^{\circ}$ due to long range reaction mechanisms.

The inelastic cross section was calculated by semiclassical Coulomb excitation theory and is shown in the inset of the figure (dashed lines). In order to describe the data, an imaginary surface term with large diffuseness $(\sim 3.5 \mathrm{fm})$ had to be introduced. This potential simulates the dynamic polarization potential accounting for the dipole couplings to the continuum, as explained before. The large breakup cross sections are about $\sim 40 \%$ of the reaction cross sections, almost twice the values found for ${ }^{9,10} \mathrm{Be}$. The contributions of elastic and non-elastic BU calculations (EBU and NEB, respectively) are investigated in [35]. In Figure 8b, it is shown the result of EBU using XCDCC calculations (red solid line), which includes the effect of the core excitation in the reaction process, and standard CDCC calculation (black dashed line) which does not. Both prescriptions produce identical results and describe well the experimental data (yellow circles), meaning that the quasielastic cross sections are not sensitive to core deformation. Although both calculations underpredict the angular distribution of ${ }^{10} \mathrm{Be}$ fragments by $\sim 20 \%$ see figure $8 \mathrm{c}$ (red solid and dashed lines), the distribution appears to be dominated by the elastic breakup mechanism. The NBU gives an important contribution (blue dot-dashed line) to the breakup yield and must be 
added incoherently to reproduce the data (TBU, black solid line). The NBU was calculated using the so-called participant-spectator IAV model [36]. Both EBU and NEB are consistent also with the energy distributions of ${ }^{10} \mathrm{Be}$ fragments. As in the previous case, Coulomb postacceleration is observed, with a kinetic energy excess of $\sim 1 \mathrm{MeV}$ giving a breakup distance of $\sim 15 \mathrm{fm}$ angleindependent, consistent with the result obtained for ${ }^{120} \mathrm{Sn}$.
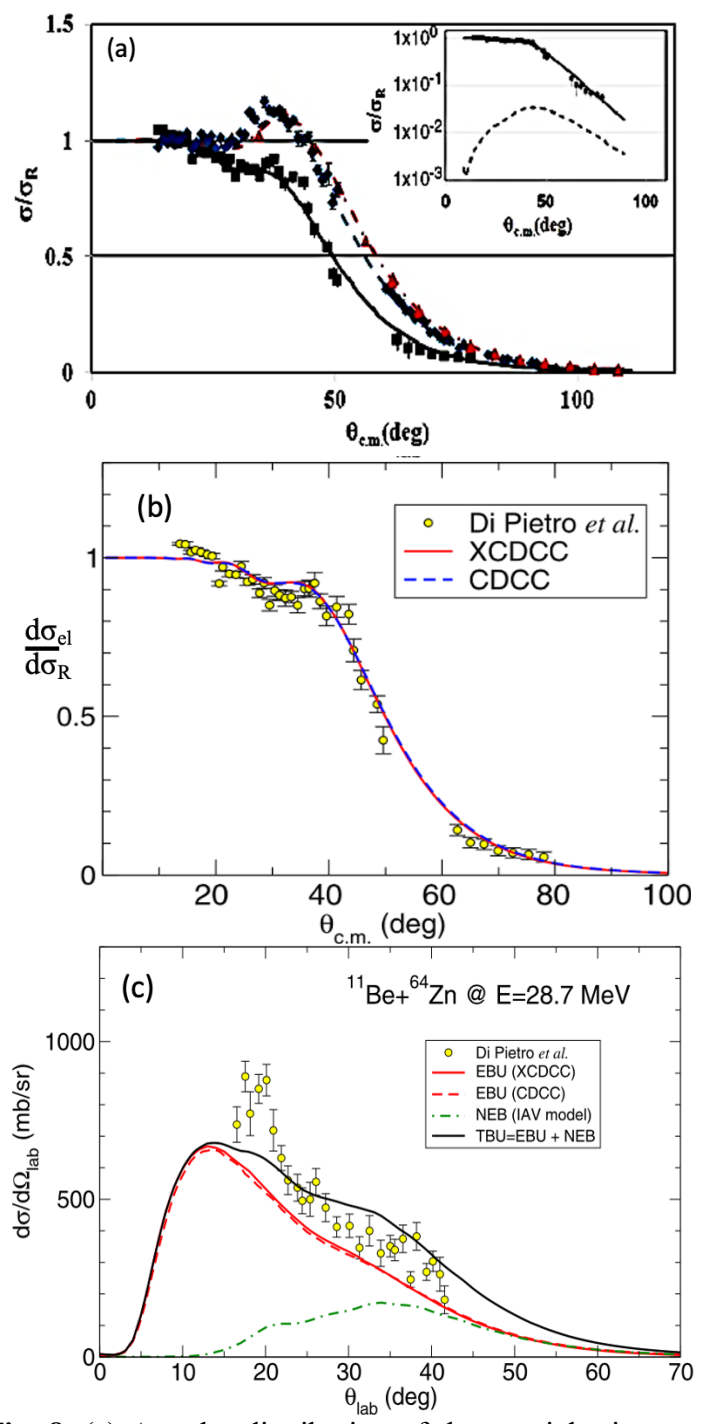

Fig. 8. (a) Angular distribution of the quasielastic cross sections for ${ }^{11} \mathrm{Be}+{ }^{64} \mathrm{Zn}$ at $\mathrm{E}=28.7 \mathrm{MeV}$ [34] (black circles), and for the system ${ }^{10,9} \mathrm{Be}+{ }^{64} \mathrm{Zn}$ at similar collision energy (blue and red squares). (b) $\mathrm{CDCC}$ and XCDCC calculations are compared with the experimental quasielastic cross section (yellow circles) [35]. (c) Angular distribution of the breakup cross sections (yellow circles). Various calculations are shown with solid, dashed and dotted lines; see text for explanation.

\section{Summary and conclusions}

This contribution intends to highlight some peculiar aspects of CBE scattering of the neutron halo systems ${ }^{6} \mathrm{He}$ (2n-halo), ${ }^{8} \mathrm{He}\left(2 \mathrm{n} / 4 \mathrm{n}\right.$-skin) and ${ }^{11} \mathrm{Be}$ (1n-halo). Common features are the large reaction and breakup cross sections, the disappearance of the Coulomb nuclear interference and the coupling to dipole states lying in the low energy continuum. Halo neutrons do not produce more fusion, as the Coulomb interaction breakups the system at distances far from the target. In the case of ${ }^{6} \mathrm{He}$ it is found a transfer mechanism of two neutrons to the continuum, whereas for ${ }^{8} \mathrm{He}$ the transfer seems to proceed through single neutron stripping to excited states in ${ }^{209} \mathrm{~Pb}$ and 4-neutron cluster transfer to ${ }^{212} \mathrm{~Pb} .{ }^{11} \mathrm{Be}$ is dominated by the breakup channel. Elastic and inelastic breakup and core deformation are essential ingredients.

Systematic studies of reactions cross sections induced by halo nuclei at several energies around the Coulomb barrier are useful to understand the dynamical behaviour, extract scaling laws and universal rules. Simpler three body models can describe the gross properties of the scattering, but few-body models are needed for accurate descriptions and to probe the nature of nucleon-nucleon correlations, often leading to new interesting discoveries.

\section{References}

1. I. Tanihata et al., Phys. Rev. Lett., 55, 2676 (1985); P.G. Hansen and B. Johnson, Europhys. Lett 4, 409 (1987)

2. T. Aumann, et al., Eur. Phys. J. A 26, 441 (2005)

3. N. Keeley et al. Prog. Part. Nucl. Phys. 63, 396 (2009)

4. A.M. Sánchez-Benítez, et al. Nucl. Phys. A 803, 30 (2008)

5. L. Acosta et al., Phys. Rev. C 84, 044604 (2011)

6. J. Cook. Nucl. Phys. A 388, 153-172 (1982)

7. M. V. Andrés and J. Gómez-Camacho. Phys. Rev. Lett. 82, 1387 (1999)

8. N. Keeley et al., Phys. Rev. C 68, 054601 (2003).

9. K. Rusek et al., Phys. Rev. C 72, 037603 (2005)

10. D. Escrig et al, Nucl. Phys. A 79, 2 (2007)

11. V. Parkar et al., Acta Phys. Pol. B 42, 761 (2011)

12. J.A. Lay et al., Phys. Rev. C 82, 024605 (2010)

13. M. Rodríguez-Gallardo et al., Phys. Rev. C 77, 064609 (2008)

14. R.S. Machintosh and N. Keley, Phys. Rev. C 79 (2009) 014611

15. N. Keeley, K.W. Kemper, K. Rusek. Phys. Rev. C 88, 017602 (2013)

16. A.M. Moro, et al, Phys. Rev. C 75, 064607 (2007)

17. M. Rodríguez-Gallardo et al., Phys. Rev. C 80, 051601(R)(2009)

18. V. Morcelle et al, Phys. Lett. B 732, 228 (2014)

19. E.F. Aguilera et al., Phys. Rev. C 83, 021601(R) (2011)

20. C. Y. Wong, Phys. Rev. Lett. 31, 766 (1973) 
21. J.J. Kolata and E.F. Aguilera et al., Phys. Rev. C 83, 027603 (2009)

22. A.M. Sánchez-Benítez et al., Acta Phys. Pol. B 37 (2006) 1 .

23. I. Martel, et al. EPJ Web of Conferences 17, 13002 (2011)

24. R. Wolski et al., Eur. Phys. Jour. A 47, 111 (2011)

25. G. Marquínez-Durán et al., Phys. Rev. C 94, 064618 (2016), Phys. Rev. C 95, 024602 (2018)

26. I. Martel et al., Phys. Rev. C 102, 34609 (2020)

27. D. J. Millener et al, Phys. Rev. C 28, 497 (1983)

28. C. Signorini et al., Nucl. Phys. A 735, 329 (2004)

29. M. Mazzocco et al., Eur. Phys. J. A 28, 295 (2006)

30. L. Acosta et al., Eur. Phys. J. A 42, 461 (2009)

31. A. Arazi et al., Phys. Rev. C 97, 044609 (2018)

32. K. Rusek et al. Act. Phys. Pol. B. 43, 129 (2012)

33. L. Acosta. PhD Thesis, University of Huelva, unpublished (2010)

34. A. Di Pietro et al. Phys. Rev. Lett. 105, 022701 (2010)

35. A. Di Pietro et al., Phys. Lett. B 798, 134954 (2019)

36. M. Ichimura, N. Austern, C.M. Vincent, Phys. Rev. 32, 431 (1985) 